\title{
Identification of a novel frameshift heterozygous deletion in exon 8 of the PAX6 gene in a pedigree with aniridia
}

\author{
OZLEM GIRAY BOZKAYA ${ }^{1}$, ESRA ATAMAN ${ }^{2}$, OZGE AKSEL KILICARSLAN ${ }^{2}$, \\ TUFAN CANKAYA ${ }^{2}$ and AYFER ULGENALP ${ }^{2}$ \\ ${ }^{1}$ Department of Pediatrics, Division of Pediatric Genetic Diseases; ${ }^{2}$ Department of Medical Genetics, \\ Faculty of Medicine, Dokuz Eylul University, 35340 Izmir, Turkey
}

Received October 1, 2015; Accepted February 4, 2016

DOI: $10.3892 / \mathrm{mmr} .2016 .5467$

\begin{abstract}
Aniridia is a congenital, panocular abnormality which is characterized by partial or complete absence of iris and various degrees of iris hypoplasia. Mutations in the PAX6 gene are found in $\sim 90 \%$ of cases with aniridia. The human PAX6 gene is located at chromosome 11p13 and encodes a transcriptional regulator that has crucial roles in the development of the eyes, central nervous system and pancreatic islets. The present study performed a clinical and genomic analysis of two families containing multiple cases of aniridia. All exons of the PAX6 gene of the probands were sequenced using the Sanger sequencing technique. A heterozygous non-stop mutation in exon 14 was identified in the first family, which has been previously reported for a different ophthalmological pathology. This mutation causes on-going translation of the mRNA into the 3'-untranslated region. In the second family, a novel frameshift heterozygous deletion in exon 8 was identified.
\end{abstract}

\section{Introduction}

Aniridia is characterized by partial or complete absence of the iris and iris hypoplasia. It is a rare congenital disorder with a frequency of 1 in 64,000-96,000 newborns. Aniridia may occur isolated or as part of a syndrome, such as 'Wilms tumor, Aniridia, Genitourinary abnormalities, Retardation' (WAGR), Rieger syndrome and Peters syndrome. $85 \%$ of patients with aniridia have an autosomal-dominant inheritance pattern (1).

Approximately $90 \%$ of patients with aniridia have mutations in the human PAX6 gene, a member of the paired box gene family. It is located on chromosome 11p13 and contains 14 exons. The PAX6 gene encodes a transcriptional regulator

Correspondence to: Professor Ozlem Giray Bozkaya, Department of Pediatrics, Division of Pediatric Genetic Diseases, Faculty of Medicine, Dokuz Eylul University, 1606 Inciralti, 35340 Izmir, Turkey

E-mail: ozlem.giray@deu.edu.tr

Key words: aniridia, congenital cataract, eye development, PAX6 gene, Sanger sequencing, non-stop mutation, novel mutation that has crucial roles in the development of the eye, central nervous system and pancreatic islets $(1,2)$.

Non-sense mutations or deletions leading to complete absence of the PAX6 protein primarily cause aniridia, while missense mutations of this gene are associated with other ocular phenotypes such as Rieger anomaly, cataract or glaucoma (3).

The present study performed a clinical and genetic analysis of two families containing several cases of aniridia. The heterozygous deletion in exon 8 , which was identified in one of the families, is a novel PAX6 mutation.

\section{Materials and methods}

Patients.Two Turkish families wereenrolled in the present study, each including five affected and 12 unaffected members, whose health status was confirmed by ophthalmological examination. Clinical data were collected and detailed family surveys were performed. Written informed consent was obtained from every adult patient and from the parents of all patients under the age of 18 . The study was approved by the ethics committee of Dokuz Eylul University (Izmir, Turkey).

DNA isolation and molecular analysis. Genomic DNA from the peripheral blood lymphocytes of all individuals were extracted using the QIAamp DNA Blood Mini kit (Qiagen, Hilden, Germany) using standard procedures. Concentration and purity of isolatedDNA were determined using a NanoDrop spectrometer (Thermo Fisher Scientific, Inc., Waltham, MA, USA). All coding exons and exon-intron boundaries of the PAX6 gene were amplified using polymerase chain reaction (PCR) with the HelixAmp ${ }^{\mathrm{TM}}$ Ready-2X-Multiplex version 2.0 PCR mix (NanoHelix, Upper Heyford, UK). Each reaction mixture contained $12.5 \mu \mathrm{l}$ master mix, $2 \mu \mathrm{l}$ distilled water, $0.5 \mu \mathrm{l}$ forward primer, $0.5 \mu \mathrm{l}$ reverse primer and DNA (20-30 ng). Primers were synthesized by Metabion (Planegg, Germany) and are listed in Table I. PAX6 gene regions are listed in Table I. Amplifications were performed using an ABI GeneAmp ${ }^{\circledR}$ PCR System 9700 thermal cycler (Applied Biosystems; Thermo Fisher Scientific, Inc.) with the following thermocycling conditions: Initial denaturation at $94^{\circ} \mathrm{C}$ for $15 \mathrm{~min}$, followed by 35 cycles of denaturation at $94^{\circ} \mathrm{C}$ for $30 \mathrm{sec}$, annealing at $57^{\circ} \mathrm{C}$ for $45 \mathrm{sec}$ (or $59^{\circ} \mathrm{C}$ for 
Table I. Primers of the PAX6 gene exons.

\begin{tabular}{llll}
\hline Exon & \multicolumn{1}{c}{ Forward primer } & Reverse primer & Amplicon length (bp) \\
\hline 4 & 5'-TTGGGAGTTCAGGCCTACCT-3' & 5'-CCAGTATCGAGAAGAGCCAAG-3' & 324 \\
5 & 5'-TCTTCTTCCTCTTCACTCTGC-3' & 5'-TGAAAGAGATAGGGAAGGATG-3' & 392 \\
$6-7$ & 5'-ATTTATCTACTTCGTTTTGATGC-3' & 5'-AGAGGGTGGGAGGAGGTAAAG-3' & 633 \\
8 & 5'-AGCTGAGATGGGTGACTGTGT-3' & 5'-AAGGGATGCACATATGGAGAG-3' & 281 \\
9 & 5'-GGGAATGTTTTGGTGAGGCT-3' & 5'-ACCTCCAACCAATTCCCTTTA-3' & 698 \\
10 & 5'-GGAACCAGTTTGATGCACAGT-3' & 5'-GCAGCAGAGCATTTAGCAGAC-3' & 302 \\
$11-12$ & 5'-GTCTGCTAAATGCTCTGCTGC-3' & 5'-AGCTCTCAAGGGTGCAGACAC-3' & 579 \\
13 & 5'-TGGCTGTGTGATGTGTTCCTC-3' & 5'-AGAAAACTTGCAGTCTCAGGC-3' & 462 \\
14 & 5'-CCATGTCTGTTTCTCAAAGGGA-3' & 5'-CCCCAGTGGTACAATACAGGA-3' & 295 \\
\hline
\end{tabular}

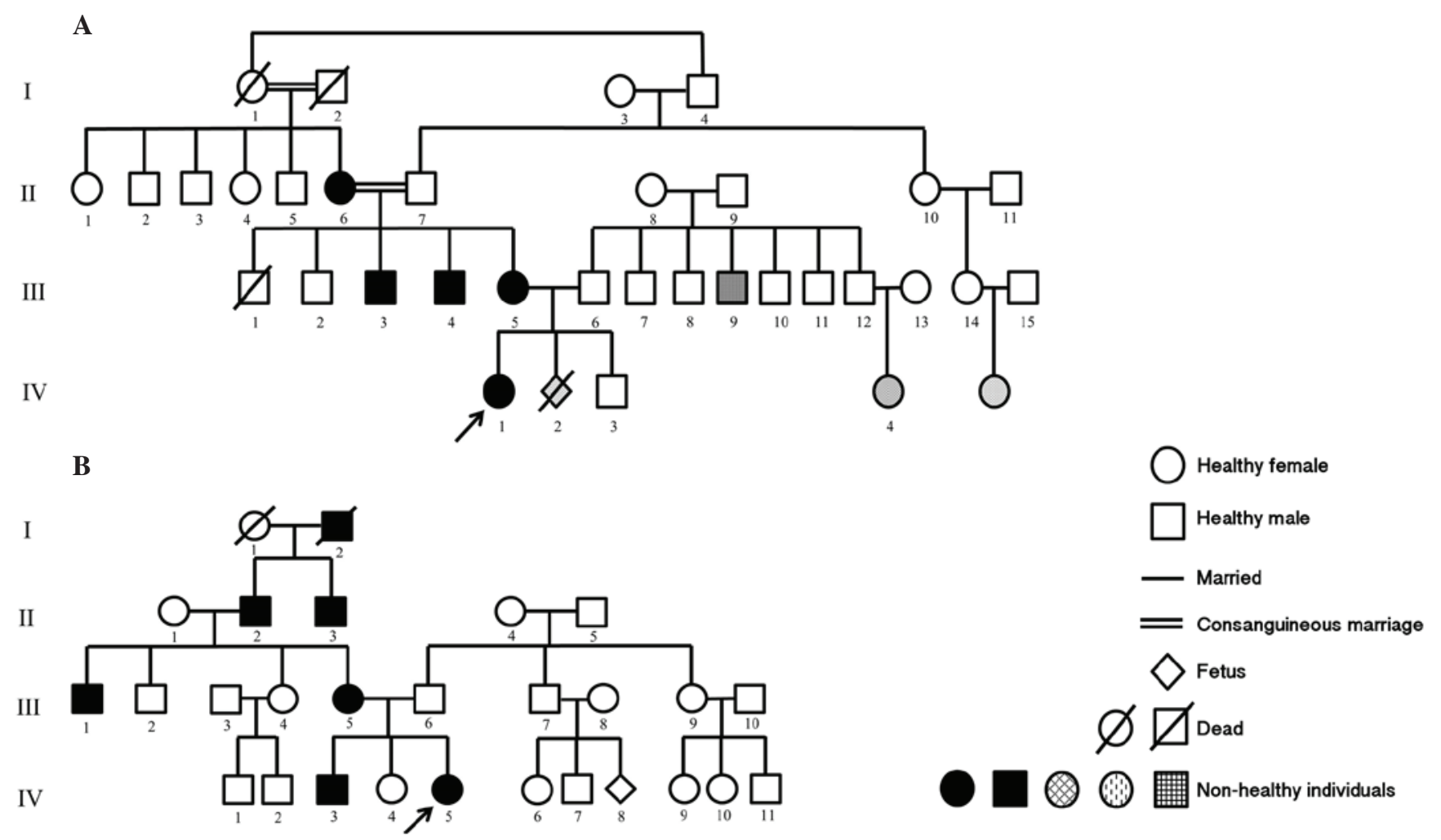

Figure 1. Pedigrees of the two families examined in the present study. (A) Family 1. II-6, III-3, III-4, III-5 and IV-1: Aniridia, congenital cataract; III-9: Stutter; IV-2: Hydrocephalus, cleft lip-palate; IV-4: Congenital heart disease. (B) Family 2. III-1, III-5, IV-3 and IV-5: Aniridia, congenital cataract. The arrows indicate the main probands. Circle, female; Square, male.

$45 \mathrm{sec}$ for exon 4) and extension at $72^{\circ} \mathrm{C}$ for $45 \mathrm{sec}$, followed by a final extension at $72^{\circ} \mathrm{C}$ for $7 \mathrm{~min}$. PCR products were verified by $2 \%$ agarose gel electrophoresis and ethidium bromide staining. Accomplished amplicons were continued for second PCR using the BigDye Terminator v3.1 Cycle Sequencing kit (Applied Biosystems). Second PCR conditions were as follows: Initial denaturation step at $96^{\circ} \mathrm{C}$ for $1 \mathrm{~min}$, followed by 25 cycles including denaturation at $96^{\circ} \mathrm{C}$ for $10 \mathrm{sec}$, annealing at $50^{\circ} \mathrm{C}$ for $5 \mathrm{sec}$ and extension at $60^{\circ} \mathrm{C}$ for 4 min. After purification using the Zymo Research purification kit (Zymo Research Corp, Irvin, CA, USA), samples were analyzed using ABI 3100 and ABI 3130 capillary electrophoresis systems (Applied Biosystems). The sequences were evaluated using the CLC Genomics Workbench 3 sequencing software (Qiagen). The Ensembl database (www. ensembl.org; GRCh38.p3, GCA_000001405.18) with the transcript ID ENST00000419022 of the PAX6 gene was used to compare the individual sequences with the reference sequence taken from the Ensembl database. All variations were analyzed using mutation and single nucleotide polymorphism (SNP) databases [Human Genome Mutation Database (http://www.hgmd.cf.ac.uk), National Center for Biotechnology Information/SNP (http://www.ncbi.nlm. nih.gov/snp), Ensembl and Mutation Taster (http://www. mutationtaster.org/)]. Each variation was confirmed by bidirectional sequencing. Variations were described according to the nomenclature recommended by the Human Genomic Variation Society (http://www.hgvs.org/mutnomen/). 
A

PAX6 gene, exon 14 Reference sequence J $^{3}$ UTR

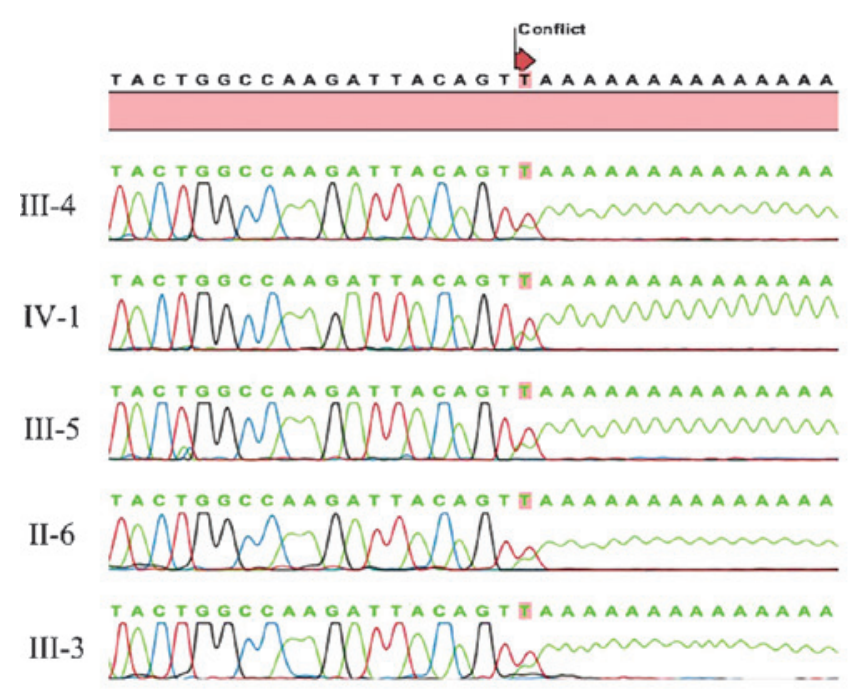

C

PAX6 gene, exon 8 Reference sequence

TGGGGCACCCGCCCIGGTTGGIATCCGGGGACTICGGTS

GGTATCC or TATCCGG del

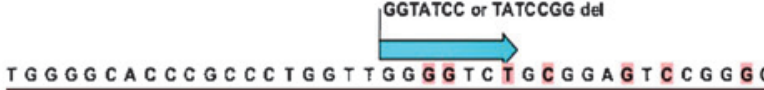

TGGGGCACCCGCCCTGGTTGGG TC IGGGA GTCGGTC III-5

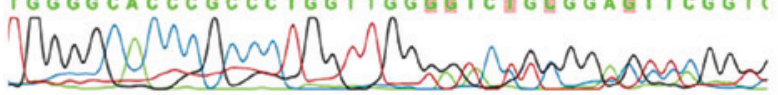

IV-3

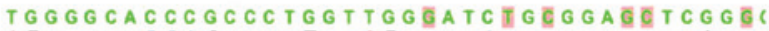

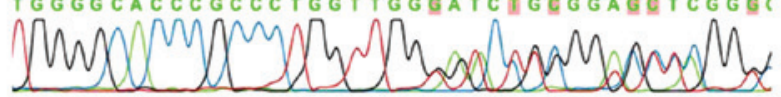

IV-5

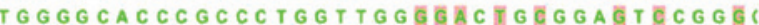

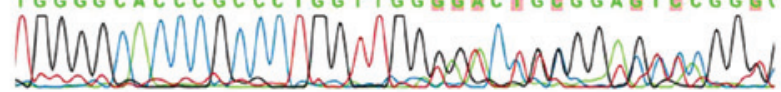

II-2
B

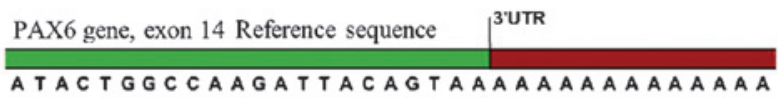

ATACTGGCCAAGATTACAGTAAAAAAAAAAAAAA

IV-3

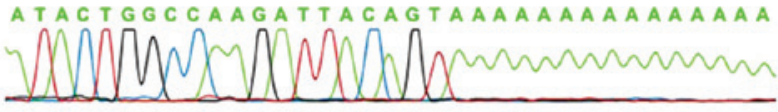

III-2

ATACTGGCCAAGATTACAGTAAAAAAAAAAAAA

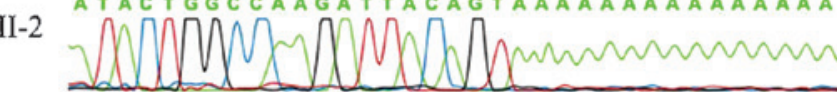

III-6

D

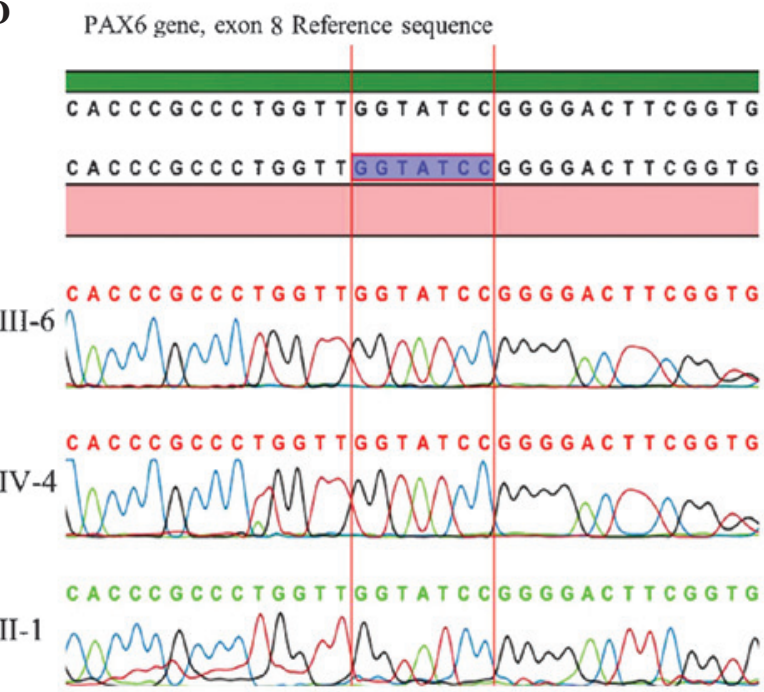

Figure 2. Partial genome sequences of the PAX6 gene of (A) affected and (B) unaffected members of Family 1 as well as (C) affected and (D) unaffected members of Family 2. UTR, untranslated region; del, deletion.

\section{Results}

First family comprised a female patient (age, three months) with bilateral congenital cataracts and partial aniridia as well as her affected mother, who were referred to the Department of Pediatrics (Izmir, Turkey) for genetic evaluation of aniridia. The mother's first child (male aged 2.5 years old), was healthy and her second pregnancy had been terminated due to cleft palate and hydrocephaly of the fetus. The patient's brother, parents, all uncles and grandparents were examined. The mother, two uncles and grandmother (on mother's side) presented with bilateral congenital cataracts and partial aniridia (Fig. 1A).

The second family included a female patient (nine years old) with bilateral congenital cataracts and aniridia. Her parents, all siblings and grandparents were assessed.
Her mother, brother (7 years old), grandfather and uncle presented with bilateral congenital cataracts and aniridia and her father suffered from loss of vision, as a result of glaucoma (Fig. 1B).

Karyotype analysis of the patients and their parents from peripheral blood lymphocytes yielded normal results. Mutation screening in all coding exons of PAX6 were performed using the Sanger sequencing technique. In the first family, a heterozygous non-stop mutation in exon 14 (c.1290 A>T, X437L) was identified in all affected members and was inherited in an autosomal dominant inheritance pattern.(Fig. 2A). Conversely, this mutation was not identified in the unaffected members.

A novel heterozygous deletion in exon 8 (c.529_535 TATCCGGdel or c.527_533 GGTATCCdel) that causes a frameshift was identified in all affected members of the second family (Fig. 2B), except for the male patient with loss 
of vision due to glaucoma. Unaffected members of the family did not have this mutation. To the best of our knowledge, the present study was the first to report on this deletion mutation in the PAX6 gene patients with aniridia.

\section{Discussion}

The present study presented two pedigrees with two different heterozygous PAX6 mutations that cause aniridia and congenital cataracts. The affected members of the first family carried a heterozygous non-stop mutation in exon 14 that causes on-going translation of mRNA into 3'-untranslated region. The mutation identified in the second family was a heterozygous deletion in exon 8 causing a frameshift, which has not been reported previously.

Aniridia is a rare congenital panocular disorder with a frequency of 1 in 64,000-96,000 newborns and is characterized by partial or complete absence of the iris and verying degrees of iris hypoplasia. It may be associated with other ocular abnormalities, including foveal hypoplasia, cataracts, nystagmus, glaucoma, optic nerve hypoplasia, ectopia lentis and keratopathy. Cataracts have been reported in 50-85\% of cases with aniridia (1). Congenital cataracts were identified in affected members of each of the two families.

Aniridia may occur in a familial or sporadic form. Approximately two-thirds of cases with aniridia have an affected parent and the inheritance pattern of familial aniridia is known to be almost exclusively autosomal dominant.

Aniridia can be an isolated disorder or part of a syndrome, such as WAGR, Rieger syndrome or Peters syndrome $(1,2)$. WAGR syndrome is a contiguous gene deletion syndrome that is caused by constitutional deletions in 11p13 leading to loss of one copy of PAX6 and WT (1). Approximately 90\% of cases with aniridia carry mutations in the human PAX6 gene. Two-thirds of all aniridia cases have intragenic mutations, while chromosomal re-arrangements have been identified in one-third of cases (2).

Mutations in the PAX6 gene, a member of the paired box gene family, have been reported as a cause of aniridia in the early 1990s $(3,4)$. The human PAX6 gene is located at chromosome $11 \mathrm{p} 13$ and consists of 14 exons encoding a transcriptional regulator protein. PAX6 has crucial roles in the morphogenesis of the eye, the central nervous system and pancreatic islets $(1,2)$. Lack of the homolog PAX6 gene causes an eyeless phenotype in Drosophila and in rats. Complete loss of the function of PAX6 can cause anophthalmia, nasal hypoplasia and prenatal death as a result of central nervous system defects in vertebrates, while PAX6 haploinsufficiency is associated with the pathologies including aniridia, foveal hypoplasia and Peters anomaly (4).

The PAX6 gene contains a serine, proline and threonine-rich trans-activation domain and two DNA-binding domains; a paired box (PD) and a homeobox (HD) that bind to each other via a glycine-rich linker region (5). PD consists of two different DNA-binding sub-domains: The N-terminal sub-domain (NTS) and C-terminal sub-domain (CTS). The NTS region has a primary role in DNA-binding. As NTS and CTS have different effects on the transactivation of HD, missense mutations in these regions may lead to distinct pathologies (1).
To date, 404 unique PAX6 gene variants have been reported (http://lsdb.hgu.mrc.ac.uk/home.php?select_db=PAX6) and $>90 \%$ of them are known/likely pathogenic mutations (2). The types of disease-associated mutations found in the patients are non-sense mutations, splicing mutations, frame-shifting insertions or deletions, in-frame insertions or deletions, missense mutations and run-on mutations. Mutations that introduce a premature termination codon into the open reading frame are predominantly associated with aniridia; while non-aniridia phenotypes are typically associated with missense mutations (6). The classical pathology of aniridia is found in individuals carrying a mutation that introduces a premature termination codon, while missense mutations frequently cause non-aniridia pathologies, including cataracts, glaucoma, optic nerve hypoplasia, foveal hypoplasia, Peters anomaly or microphthalmia $(2,6)$.

C-terminal extensions (CTE) or run-on mutations are caused by a change in the termination codon to an amino acid codon, therefore leading to uninterrupted translation into the $3^{\prime}$ untranslated region $(2,4,6)$. Hingorani et al (7) reported that patients carrying CTE mutations have more severe pathologies than others. In the present study, a heterozygous non-stop mutation in exon 14 (c.1290 A>T, X437L) was identified in the first family. This mutation was first reported by Baum et al (8) in a female patient (age, three months) with poor vision, absence of the inner margin of the iris and nystagmus.

In the present study, a female patient with aniridia carrying the heterozygous non-stop mutation in exon 14 (c.1290 A>T, $\mathrm{X} 437 \mathrm{~L}$ ) had a pregnancy termination due to cleft palate and hydrocephaly of the fetus; however, genetic analysis of the fetus could not be performed. Takagi et al (9) reported on a young female patient with a heterozygous 310-kb deletion of the downstream flanking region of PAX6, isolated $\mathrm{GH}$ deficiency, cleft palate and bilateral optic disc cupping. It was indicated that PAX6 is involved in palate development through Sonic hedgehog.

Of note, the present study identified a novel heterozygous deletion in exon 8 (c.529_535 TATCCGGdel or c.527_533 GGTATCCdel) causing a frameshift in all affected members of the second family, except for the male patient with loss of vision due to glaucoma. To the best of our knowledge, the present study was the first to report on this frameshift deletion. Further studies are required to define the phenotype associated this deletion.

In conclusion, the present study identified a heterozygous deletion and a run-on mutation in PAX6 in two families with autosomal dominant aniridia. In one of the families, a novel frameshift heterozygous deletion in exon 8 was identified. It is recommended that cases with aniridia refer to genetics departments for clinical assessment and genetic counselling.

\section{References}

1. Kokotas $\mathrm{H}$ and Peterson MB: Clinical and molecular aspects of aniridia. Clin Genet 77: 409-420, 2010.

2. Hingorani M, Hanson I and van Heyningen V: Aniridia. Eur J Hum Genet 20: 1011-1017, 2012.

3. Axton R, Hanson I, Danes S, Sellar G, van Heyningen V and Prosser J: The incidence of PAX6 mutation in patients with simple aniridia: An evaluation of mutation detection in 12 cases. J Med Genet 34: 279-286, 1997. 
4. Lee HJ and Colby KA: A review of the clinical and genetics aspects of aniridia. Semin Ophthalmol 28: 306-312, 2013.

5. Singh S, Chao LY, Mishra R, Davies J and Saunders GF: Missense mutation at the C-terminus of PAX6 negatively modulates homeodomain function. Hum Mol Genet 10: 911-918, 2001

6. Tzoulaki I, White IM and Hanson IM: PAX6 mutations: Genotype-phenotype correlations. BMC Genet 6: 27, 2005.

7. Hingorani M, Williamson KA, Moore AT and van Heyningen V: Detailed ophthalmologic evaluation of 43 individuals with PAX6 mutations. Invest Ophthalmol Vis Sci 50: 2581-2590, 2009.
8. Baum L, Pang CP, Fan DS, Poon PM, Leung YF, Chua JK and Lam DS: Run-on mutation and three novel nonsense mutations identified in the PAX6 gene in patients with aniridia. Hum Mutat 14: 272-273, 1999.

9. Takagi M, Nagasaki K, Fujiwara I, Ishii T, Amano N, Asakura Y, Muroya K, Hasegawa Y, Adachi M and Hasegawa T: Heterozygous defects in PAX6 gene and congenital hypopituitarism. Eur J Endocrinol 172: 37-45, 2015. 